\title{
Granulomatous angiitis leading to a pulmonary veno-occlusive disease-like picture
}

\author{
N. Dwyer*, R. Smith\#, D. Challis”, D. Reid ${ }^{\text {I }}$ and D. Kilpatrick*
}

ABSTRACT: Pulmonary veno-occlusive disease (PVOD) is a rare cause of pulmonary hypertension characterised by extensive fibrotic occlusion of pulmonary veins. PVOD has a similar insidious presentation to idiopathic pulmonary arterial hypertension but responds poorly to conventional therapies and has a worse prognosis.

The current study reports the case of a Caucasian female with a long history of progressive dyspnoea ultimately diagnosed as focal granulomatous venulitis leading to a pulmonary venoocclusive disease-like pathology. The present study highlights the challenges in diagnosing and treating this condition.

KEYWORDS: Acute pulmonary oedema, bosentan, granulomatous venulitis, infliximab, pulmonary veno-occlusive disease, sildenafil

\section{CASE REPORT}

A 43-yr-old female was extensively investigated because of progressive exercise induced fatigue and breathlessness without wheeze or cough. The patient was otherwise well. She ceased smoking at the time of symptom onset, having accumulated a 25 pack-yr smoking history. There was no relevant family history or occupational and drug exposures. There were no abnormal examination findings early in the natural history of the disease. Arterial oxygen saturation $\left(\mathrm{Sa}_{2} \mathrm{O}_{2}\right)$ at rest was $97 \%$ on room air.

A chest radiograph (CXR), electrocardiogram, echocardiogram and myocardial thallium scan were all unremarkable. Lung function tests demonstrated a mild degree of airflow obstruction with no reversibility: forced expiratory volume in one second (FEV1) $2.15 \mathrm{~L}$ (85\% predicted); forced vital capacity (FVC) $2.84 \mathrm{~L}$ (94\% pred); FEV1/FVC ratio $76 \%$; mean forced expiratory flow between 25 and $75 \%$ FVC $61 \%$ pred; diffusing capacity of the lung for carbon monoxide (DL,CO) $94 \%$ pred. A histamine challenge showed a provocation concentration causing a fall in FEV1 of $20 \%$ of $0.825 \mathrm{mg} \cdot \mathrm{mL}^{-1}$, which is consistent with severe airway hyperresponsiveness. A provisional diagnosis of asthma prompted a 3-week trial of oral prednisolone and introduction of a corticosteroid/long-acting $\beta_{2}$-agonist combination inhaler device. This intervention was ineffective and did not improve lung function tests. A cardiopulmonary exercise test was aborted due to severe fatigue at $87 \%$ of predicted workload. The maximal oxygen uptake $\left(V^{\prime} \mathrm{O}_{2}\right.$, $\left.\max \right)$ was $93 \%$ of predicted with no exercise induced fall in $\mathrm{SaO}_{2}$. The patient was then lost to follow-up.

The patient re-presented with worsening breathlessness and tender, erythematous nodules on her legs consistent with a diagnosis of erythema nodosum 3 yrs later. The cutaneous lesions resolved spontaneously without any treatment. A ventilation/perfusion $\left(V^{\prime} / Q^{\prime}\right)$ scan was normal. High-resolution computed tomography (HRCT) of the lungs showed mild expiratory gas trapping with patchy peripheral bronchocentric opacities and minor septal thickening, but no mediastinal lymphadenopathy. Her serum biochemistry and full blood count were normal, erythrocyte sedimentation rate (ESR) was elevated at $41 \mathrm{~mm} \cdot \mathrm{h}^{-1}$ (normal rate $<15 \mathrm{~mm} \cdot \mathrm{h}^{-1}$ ) and angiotensin-converting enzyme level was $21.5 \mathrm{IU} \cdot \mathrm{L}^{-1}$ (normal range $8.3-21.4 \mathrm{IU} \cdot \mathrm{L}^{-1}$ ). The patients' serum antinuclear (ANA) and antineutrophil cytoplasmic (ANCA) antibody titres were negative. A diagnosis of pulmonary sarcoid was
AFFILIATIONS

*Depts of Cardiology,

\#Anatomical Pathology, and

"Respiratory Medicine, Royal Hobart Hospital

Tasmania, Australia.

CORRESPONDENCE

N. Dwyer

Cardiology Dept

Royal Hobart Hospital

48 Liverpool St

Hobart

Tasmania

Australia 7000

Fax: 6162342852

E-mail: ndwyer@utas.edu.au

Received:

January 032008

Accepted after revision:

September 042008

STATEMENT OF INTEREST

Statements of interest for D. Reid and

D. Kilpatrick can be found at

www.erj.ersjournals.com/misc/

statements.shtml 
considered but endobronchial and transbronchial biopsies along with bronchoalveolar lavage were unremarkable. Without a satisfactory explanation of her symptoms, the patient was lost to follow-up for $1 \mathrm{yr}$.

The patient continued to complain of severe exertional breathlessness and fatigue and ceased full-time employment. She developed acute onset of left-sided pleuritic chest pain and increased breathlessness 4 yrs after initial presentation. A CXR revealed a $2-\mathrm{cm}$ area of focal consolidation in the left lung-mid zone. Liver and renal function tests and full blood count were all normal but her ESR was elevated at $73 \mathrm{~mm} \cdot \mathrm{h}^{-1}$. The patient had a positive ANA titre at 1:40 with a nucleolar pattern. Antiglomerular basement membrane antibody titres and perinuclear-staining- and cytoplasmic-staining-ANCA were all normal. Her urine microscopy was normal and urinary calcium excretion was $9.8 \mathrm{mmol} \cdot 24 \mathrm{~h}^{-1}$ (normal range 2$\left.8 \mathrm{mmol} \cdot 24 \mathrm{~h}^{-1}\right)$. Arterial blood gases demonstrated hypoxaemia: $\mathrm{pH} 7.46$; carbon dioxide arterial tension $\left(\mathrm{Pa}, \mathrm{CO}_{2}\right)$ $31 \mathrm{mmHg}$; arterial oxygen tension $\left(\mathrm{Pa}_{\mathrm{a}} \mathrm{O}_{2}\right) 64 \mathrm{mmHg}$; and $\mathrm{HCO}_{3} 20.8 \mathrm{mmol}$. The uncertain diagnosis and progression of symptoms prompted a thoracoscopic lung biopsy.
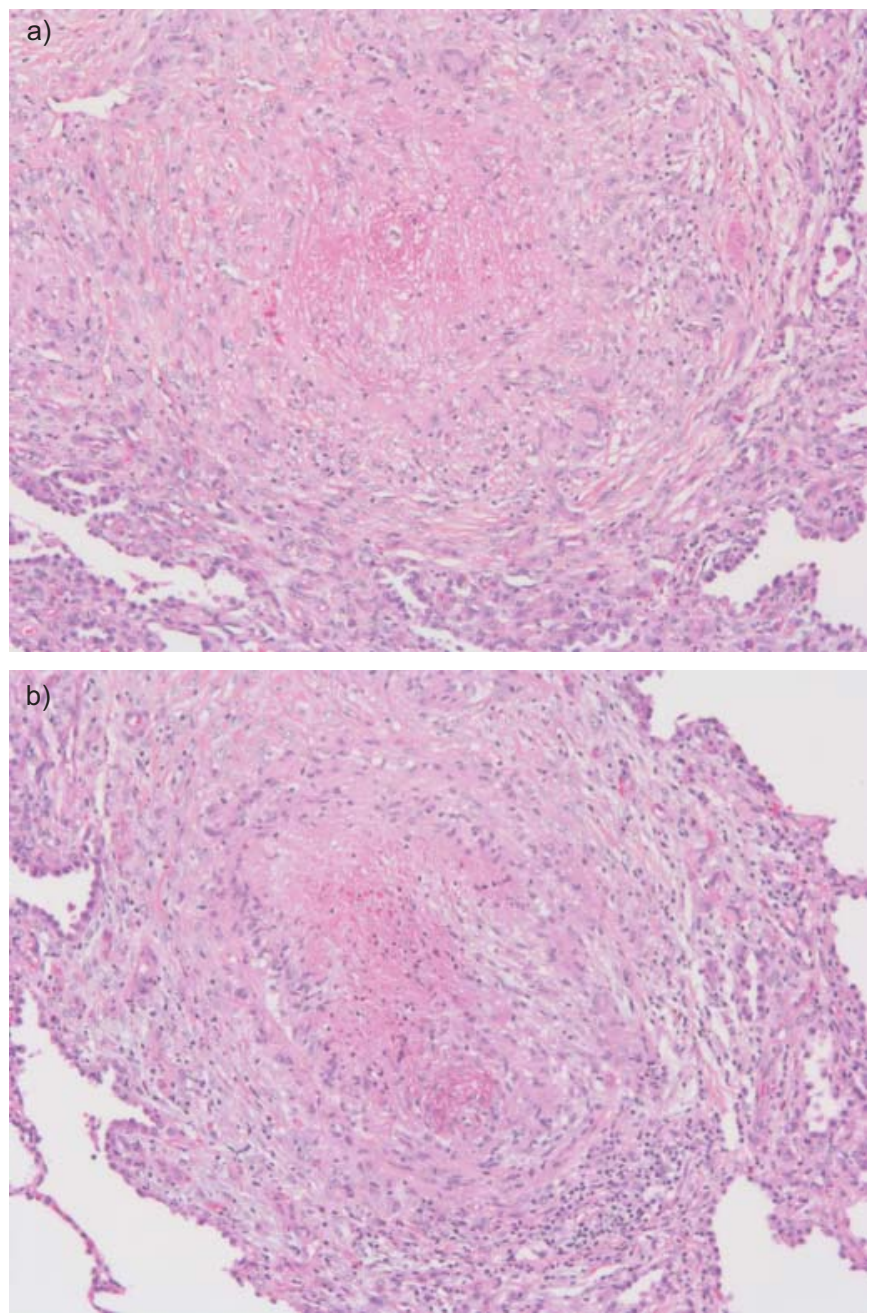

FIGURE 1. a, b) Histological examination showing occlusive intravascular granulomas with necrosis (haematoxylin and eosin stain).
Histological examination of the lung biopsies showed intravascular granulomas, some of which contained central necrosis (fig. 1), as well as sparse interstitial and peribronchial granulomas. Routine culture of the lung biopsies was negative for Mycobacteria. Widespread fibro-intimal changes in the walls of small venules were present, causing luminal obliteration with the development of dilated collateral vascular channels in the visceral pleura and interlobular septa. In some areas, obliterated and recanalised veins were located in areas of collagenous scarring (fig. 2). Areas of haemorrhagic infarction and subpleural scarring were present that were thought to be a further consequence of the vasculopathy.

National and international opinions on the pathological diagnosis and management were sought. Opinions differed, with some favouring a diagnosis of necrotising sarcoid granulomatosis, whilst others argued that the relative paucity of extravascular granulomata and the extensive fibro-intimal thickening and occlusive venulopathy favoured a diagnosis of pulmonary veno-occlusive disease (PVOD), possibly secondary to focal granulomatous venulitis.

A diagnosis of sarcoid was considered most likely and the patient was started on prednisolone $40 \mathrm{mg}$ daily and weekly oral methotrexate. Given the prominent vasculitis and thrombosis, she was anti-coagulated with warfarin. This regimen provided little symptomatic benefit after 6 months, but there was apparent stabilisation of disease progression with some improvement in gas exchange as measured by arterial blood gases: $\mathrm{pH}$ 7.45; $\mathrm{Pa}_{2} \mathrm{CO}_{2} 34 \mathrm{mmHg} ; \mathrm{Pa}_{1} \mathrm{O}_{2} 72 \mathrm{mmHg}$; and $\mathrm{HCO}_{3}$ $24 \mathrm{mmol}$.

Reduction of prednisolone below $10 \mathrm{mg}$ daily resulted in the development of recurrent episodes of migratory superficial thrombophlebitis. The patient also developed a small fibre peripheral neuropathy, which was confirmed on nerve conduction studies. Azathioprine $100 \mathrm{mg}$ daily was introduced to replace methotrexate. Prednisolone could then be ceased without recurrence of thrombophlebitis.

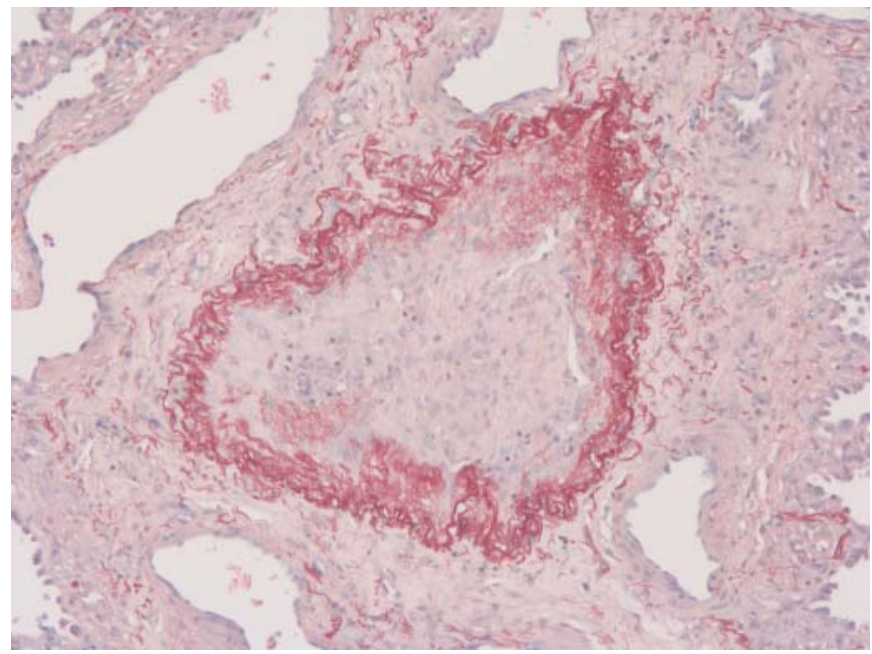

FIGURE 2. Elastic stain (orcein giemsa) demonstrating fibro-intimal obliteration of small vessels. 
Exercise testing 7 yrs after the onset of symptoms showed a fall in $\mathrm{Sa}_{1} \mathrm{O}_{2}$ from $97 \%$ to $85 \%$ at a workload $60 \%$ of the predicted maximum. Right heart studies and pulmonary angiography did not identify significant pulmonary hypertension or arterial obstruction. A trial of infliximab, an anti-tumour necrosis factor- $\alpha$ monoclonal antibody, was undertaken and resulted in an increase in exercise capacity with $V^{\prime} \mathrm{O}_{2}$, max improving from 51 to $61 \%$ after 3 months. Therefore, infliximab was continued at 12-weekly intervals. After $2 \mathrm{yrs}$, there appeared to be evidence of disease progression with $\mathrm{Sa}_{1} \mathrm{O}_{2}$ falling to $77 \%$ on room air at only $50 \%$ of maximal workload. Increasing the dosing frequency of infliximab to every 6 weeks stabilised the patients' exercise capacity and led to a minimal $\mathrm{Sa}_{2} \mathrm{O}_{2}$ of $87 \%$ at peak exercise over the next year.

Nearly 10 yrs from symptom onset there was rapid worsening of breathlessness and fatigue. After $100 \mathrm{~m}$ of slow walking, $\mathrm{Sa}_{1} \mathrm{O}_{2}$ fell to $85 \%$ on room air. A HRCT showed widespread septal thickening and ground-glass opacity. Scattered nodules were seen around the pleura and in the fissures. A gallium scan did not demonstrate active pulmonary inflammation and infliximab was ceased. A $V^{\prime} / Q^{\prime}$ scan showed patchy but extensive perfusion defects bilaterally. Right heart studies were repeated: mean pulmonary artery pressure was $38 \mathrm{mmHg}$ (normal range 9-16 $\mathrm{mmHg}$ ); pulmonary capillary wedge pressure was $13 \mathrm{mmHg}$ (normal range $1-10 \mathrm{mmHg}$ ); cardiac index was $2.6 \mathrm{~L} \cdot \mathrm{min}^{-1} \cdot \mathrm{m}^{-2}$ (normal range $2.6-$ $4.2 \mathrm{~L} \cdot \mathrm{min}^{-1} \cdot \mathrm{m}^{-2}$ ); and pulmonary vascular resistance was 5.7 Wood units (normal value $<2.0$ Wood units). Pulmonary angiography showed marked pruning of small vessels with occasional absence of segmental arteries. It was striking that some regional pulmonary architecture looked normal.

The patient was assessed for lung transplantation but her survival was considered to be better with continued medical therapy and she was started on bosentan $62.5 \mathrm{mg}$ b.d. She was admitted to hospital 16 days later and was more breathless with a history suggesting paroxysmal nocturnal dyspnoea over the previous 6 nights. Her CXR was unchanged showing widespread patchy interstitial infiltrates with basal septal lines and bilateral hilar enlargement. The symptoms settled upon cessation of bosentan for $48 \mathrm{~h}$. Rechallenge with bosentan resulted in acute respiratory distress soon after the first dose. The patients' symptoms responded to oxygen and furosemide therapy. Echocardiography showed no deterioration in left ventricular systolic function. Bosentan was permanently discontinued. A similar response was not experienced with sildenafil $50 \mathrm{mg}$ b.d., which was tolerated but ineffective. She remained extremely dyspnoeic with profound hypoxia. The patient deteriorated rapidly within 2 months, dying before a lung transplant became available.

Post mortem examination of the lungs demonstrated widespread classical changes of PVOD. There was fibromuscular obliteration of small and large calibre pulmonary veins, widespread interstitial oedema and fibrosis, and areas of congestion and infarction, but no evidence of iron or calcium encrustation of elastic fibres in the walls of veins or alveoli. There was pulmonary arterial remodelling with associated right atrial dilatation and right ventricular hypertrophy. Unlike the lung biopsy 7 years prior, there was no evidence of granulomatous inflammation.

\section{DISCUSSION}

PVOD is a clinicopathological syndrome accounting for $<10 \%$ of pulmonary hypertension cases [1]. The estimated annual incidence is 0.2 per million in the general population [2]. Little is understood of its epidemiology, aetiology, natural history and optimal treatment. Diagnosis remains difficult with a mean duration of 49 months from symptom onset to diagnosis [3]. Prognosis is poor with most patients dying within 2 years of diagnosis [4]. PVOD should be suspected in patients with pulmonary hypertension, radiographic evidence of pulmonary oedema and a normal pulmonary capillary wedge pressure.

A multitude of factors including infection, genetics, toxic exposure, thrombotic diathesis and autoimmune disorders have been implicated in the aetiology of PVOD $[5,6]$. Interestingly, granulomatous phlebitis, with or without generalised venulitis, has been reported in the setting of PVOD [710]. The present case provides indirect evidence that PVOD is the final pathological manifestation of a sustained immunological response to an initial or ongoing pulmonary insult. No evidence of granulomatous disease was present at post mortem examination, increasing the possibility that the fibromuscular obliteration of pulmonary veins was a sustained process long after the initial granulomatous inflammation had resolved.

PVOD is usually diagnosed by surgical lung biopsy. The primary pathology is obliteration of the pulmonary veins and venules by intimal fibrosis and medial hypertrophy, especially in the interlobular septae. Recanalisation of occluded vessels may occur over time, sometimes with calcium or iron encrusting venous and alveoli elastic fibres. Secondary medial hypertrophy and thrombosis of pulmonary arteries is common. Other findings may include alveolar congestion and haemorrhage with parenchymal haemosiderosis and dilated pulmonary lymphatics. Arteritis and plexiform lesions are absent. Repeated episodes of severe interstitial oedema may lead to fibrosis, which can be confused with idiopathic pulmonary fibrosis.

The radiographic findings of PVOD are often minimal. The two most typical features on HRCT are interlobular septal thickening and mosaic-pattern ground-glass attenuation, reflecting interstitial oedema. Other features include effusions and mediastinal lymphadenopathy [11]. The results of $V^{\prime} / Q^{\prime}$ scans are variable ranging from normal to high probability mismatches. Pulmonary angiograms often fail to correlate with $V^{\prime} / Q^{\prime}$ scans. This may relate to the higher pressure used to inject contrast medium, which overcomes pulmonary venous resistance in PVOD [12]. Pulmonary function tests often show a reduction in $D \mathrm{~L}, \mathrm{CO}$ with normal spirometry and lung volumes.

Therapies such as vasodilators, immunosuppressants, anticoagulants and oxygen are of minimal efficacy. Lung transplantation remains the only therapy capable of significantly prolonging life in patients with PVOD. Recurrence after lung transplantation has been reported and suggests that extrapulmonary factors may have a role in the pathogenesis of PVOD [13].

The use of inhaled nitric oxide, i.v. epoprostenol or adenosine in PVOD may lead to the development of sometimes fatal pulmonary oedema $[5,14]$. The mechanism of oedema is 
probably related to pulmonary arterial vasodilation without concomitant pulmonary venodilation, resulting in increased transcapillary hydrostatic forces and transudation of fluid into the pulmonary interstitium. Bosentan was poorly tolerated by the patient, inducing probable pulmonary oedema. Sildenafil has been tolerated in PVOD in two previous case reports [15, $16]$.

Sarcoidosis simulating PVOD has been reported only twice before $[8,17]$. Pulmonary hypertension is only seen in $5 \%$ of all sarcoidosis cases [18]. Mechanisms contributing to the development of pulmonary hypertension include the presence of vasoactive factors, granulomatous vascular involvement or extrinsic compression of pulmonary arteries by fibrosis or lymphadenopathy. There have been discordant results on the benefits of corticosteroids in patients with sarcoidosis and pulmonary hypertension [18]. Infliximab has been used successfully in refractory pulmonary sarcoid, although the effect on long-term outcome remains uncertain [19-21]. In the present patient there appeared to be disease stabilisation over a 2-yr period.

\section{Conclusion}

The diagnosis of pulmonary veno-occlusive disease is difficult but should be suspected when the degree of fatigue and dyspnoea are out of proportion to changes detected with imaging or haemodynamic and pulmonary function monitoring. The present case shows progression from a focal granulomatous angiitis without pulmonary hypertension to a pulmonary veno-occlusive disease-like pattern with features of pulmonary hypertension, raising the possibility that pulmonary veno-occlusive disease is the final pathological manifestation of pulmonary venous injury. Infliximab appeared to temporarily stabilise the disease progress. Pulmonary venoocclusive disease needs to be distinguished from other forms of pulmonary arterial hypertension as it has a poorer prognosis and response to pulmonary arterial dilators; therefore, prompting earlier consideration of lung transplantation. Pulmonary dilators should be used cautiously as a bridge to transplantation as there are distinct risks of acute and perhaps fatal pulmonary oedema.

\section{REFERENCES}

1 Burke AP, Farb A, Virmani R. The pathology of primary pulmonary hypertension. Mod Pathol 1991; 4: 269-282.

2 Rubin LJ. Current concepts: primary pulmonary hypertension. N Engl J Med 1997; 336: 111-117.

3 Lantuéjoul S, Sheppard MN, Corrin, B, Burke MM, Nicholson AG., Pulmonary veno-occlusive disease and pulmonary capillary hemangiomatosis. Am J Surg Pathol 2006; 30: 850-857.

4 Shackelford GD, Sacks EJ, Mullins JD, McAlister WH. Pulmonary venoocclusive disease: case report and review of the literature. AJR Am J Roentgenol 1977; 128: 643-648.
5 Mandel J, Mark EJ, Hales CA. Pulmonary veno-occlusive disease. Am J Respir Crit Care Med 2000; 162: 1964-1973.

6 McDonnell PJ, Summer WR, Hutchins GM. Pulmonary veno-occlusive disease. Morphological changes suggesting a viral cause. J Am Med Assoc 1981; 246: 667-671.

7 Crissman JD, Koss M, Carson RP. Pulmonary venoocclusive disease secondary to granulomatous venulitis. Am J Surg Pathol 1980; 4: 93-99.

8 Hoffstein V, Ranganathan N, Mullen JB. Sarcoidosis simulating pulmonary veno-occlusive disease. Am Rev Respir Dis 1986; 134: 809-811.

9 Schachter EN, Smith GJ, Cohen GS, Lee SH, Laser A, Gee JB. Pulmonary granulomas in a patient with pulmonary veno-occlusive disease. Chest 1975; 67: 487-489.

10 Hamada K, Teramoto S, Narita N, Yamada E, Teramoto K, Kobzik L. Pulmonary veno-occlusive disease in pulmonary Langerhans' cell granulomatosis. Eur Respir J 2000; 15: 421-423.

11 Resten A, Maitre S, Humbert M, et al. Pulmonary hypertension: $\mathrm{CT}$ of the chest in pulmonary veno-occlusive disease. AJR Am J Roentgenol 2004; 183: 65-70.

12 Bailey CL, Channick RN, Auger WR, et al. "High probability" perfusion lung scans in pulmonary venoocclusive disease. Am J Respir Crit Care Med 2000; 162: 1974-1978.

13 Izbicki G, Shitrit D, Schechtman I, et al. Recurrence of pulmonary veno-occlusive disease after heart-lung transplantation. J Heart Lung Transplant 2005; 24: 635-637.

14 Palmer SM, Robinson LJ, Wang A, Gossage JR, Bashore T, Tapson VF. Massive pulmonary oedema and death after prostacyclin infusion in a patient with pulmonary venoocclusive disease. Chest 1998; 113: 237-340.

15 Kuroda T, Hirota H, Masaki M, et al. Sildenafil as adjunct therapy to high-dose epoprostenol in a patient with pulmonary veno-occlusive disease. Heart Lung Circ 2006; 15: 139-142.

16 Barreto AC, Franchi SM, Castro CR, Lopes AA. One-year follow-up of the effects of sildenafil on pulmonary arterial hypertension and veno-occlusive disease. Braz J Med Biol Res 2005; 38: 185-195.

17 Portier F, Lerebours-Pigeonniere G, Thiberville L, et al. Sarcoidosis simulating a pulmonary veno-occlusive disease. Rev Mal Respir 1991; 8: 101-102.

18 Nunes H, Humbert M, Capron F, et al. Pulmonary hypertension associated with sarcoidosis: mechanisms, haemodynamics and prognosis. Thorax 2006; 61: 68-74.

19 Baughman RP, Lower EE. Infliximab for refractory sarcoidosis. Sarcoidosis Vasc Diffuse Lung Dis 2001; 18: 70-74.

20 Yee AMF, Pochapin MB. Treatment of complicated sarcoidosis with infliximab anti-tumor necrosis- $\alpha$ therapy. Ann Intern Med 2001; 135: 27-31.

21 Baughman RP, Drent M, Kavuru M, et al. Infliximab therapy in patients with chronic sarcoidosis and pulmonary involvement. Am J Respir Crit Care Med 2006; 174: 795-802. 